\title{
INFLUÊNCIA DO ÁCIDO INDOLBUTÍRICO NO ENRAIZAMENTO DE ESTACAS DE QUARESMEIRA
}

\author{
INDOLEBUTYRIC ACID INFLUENCE ON THE ROOTING OF Tibouchina \\ fothergillae (D. C.) Cogn.
}

\author{
Rodrigo NICKNICH ${ }^{1}$ \\ Arthur Hermann WEISER ${ }^{2}$ \\ Katia Christina ZUFFELLATO-RIBAS ${ }^{3}$
}

\begin{abstract}
RESUMO
Tibouchina fothergillae (D. C.) Cogn., popularmente conhecida por quaresmeira, é uma espécie nativa do Brasil, de grande valor paisagístico, que pode alcançar até 1,5 metros de altura. Visando a elaboração de um protocolo de enraizamento da espécie, o objetivo deste trabalho foi verificar a influência de diferentes concentrações de ácido indolbutírico (AIB) na indução radicial em estacas caulinares de quaresmeira. Em 15/04/2011 foram coletadas estacas semilenhosas de plantas matrizes localizadas nos jardins do Campus III da Universidade Federal do Paraná, as quais foram confeccionadas com $8 \mathrm{~cm}$ de comprimento mantendo-se duas folhas cortadas ao meio na porção apical. As estacas foram submetidas aos seguintes tratamentos veiculados na forma de talco: $0 \mathrm{mg} \mathrm{Kg}^{-1}, 1000 \mathrm{mg} \mathrm{Kg}^{-1}$ e $2000 \mathrm{mg} \mathrm{Kg}^{-1}$ de AIB, sendo plantadas em tubetes com vermiculita de granulometria média e casca de arroz carbonizada (1:1) como substrato e mantidas em casa de vegetação com nebulização intermitente. Foi utilizado um delineamento inteiramente casualizado, com 5 repetições e 20 estacas por unidade experimental. Após 60 dias, foram avaliadas as seguintes variáveis: porcentagem de estacas enraizadas; número de raízes por estaca; comprimento médio das três maiores raízes por estaca $(\mathrm{cm})$; porcentagem de estacas com calos, vivas e mortas. Não houve diferença significativa entre os tratamentos, tendo como maior média de enraizamento $92 \%$, para os tratamentos com 0 e $2000 \mathrm{mg} \mathrm{Kg}^{-1} \mathrm{de}$ AIB, sendo possível concluir que não é necessária a aplicação de auxina exógena no enraizamento de estacas de Tibouchina fothergillae.
\end{abstract}

Palavras-chave: Tibouchina fothergillae; auxina; paisagismo; propagação vegetativa

\section{ABSTRACT}

Tibouchina fothergillae (D. C.) Cogn. is a Brazilian native species, reaching 1,5 meters in height, with great landscape value. Aiming to develop a rooting protocol for the species, the objective of this study was to evaluate the indolebutyric acid (IBA) influence on the rooting induction of semi hardwood Tibouchina fothergillae cuttings. The shoots were collected in 15/04/2011 at the gardens of the Federal University of Paraná, Campus III, and cut to $8 \mathrm{~cm}$ in length, keeping two half leaves on the apex. The cuttings were submitted to the following treatments: $0 \mathrm{mg} \mathrm{Kg}{ }^{-1}, 1000 \mathrm{mg} \mathrm{Kg}^{-1}$ and $2000 \mathrm{mg} \mathrm{Kg}^{-1}$ of IBA in powder, planted in tubes containing medium size vermiculite and carbonized rice husk (1:1) and maintained in greenhouse with intermittent mist. Data were analyzed using a completely randomized design, with 5 replications of 20 shoots per experimental unit. After 60 days, were evaluated: the percentage of rooted cuttings; number of roots per cutting; average length of the three biggest roots per cutting $(\mathrm{cm})$; percentage of cuttings with calluses, alive and dead. The difference between the treatments was not significant, with the higher average of rooting being $92 \%$ for the treatments with 0 and 2000 $\mathrm{mg} \mathrm{Kg}^{-1}$ of IBA, leading to the conclusion that exogenous auxin is not necessary on the rooting of Tibouchina fothergillae cuttings.

Key-words: Tibouchina fothergillae; auxin; landscape; vegetative propagation

\footnotetext{
${ }^{1}$ Eng. Agr., Universidade Federal do Paraná. Curitiba, PR, Brasil. Bolsista de Iniciação Tecnológica Industrial do CNPq - Nível A. Email: rodrigonicknich@gmail.com

${ }^{2}$ Eng. Agr., Universidade Federal do Paraná. Curitiba, PR, Brasil. Bolsista de Iniciação Científica do CNPq

${ }^{3}$ Bióloga, Pós-Doutora, Departamento de Botânica, Setor de Ciências Biológicas, Universidade Federal do Paraná (UFPR), Curitiba, PR, Brasil. E-mail: kazu@ufpr.br
} 
NICKNICH, R., et al. Influência do ácido...

\section{INTRODUCÃO}

Tibouchina fothergillae (D. C.) Cogn. é uma planta da família Melastomataceae, nativa da Mata Atlântica Brasileira. Apresenta porte arbustivo, com cerca de 1,5 metros de altura e flores de coloração púrpura. Floresce e frutifica em quase todos os meses do ano, sendo os mais representativos abril e maio (Silva e Affonso, 2005) e sendo por isso conhecida popularmente por quaresmeira.

Por apresentar belíssimas flores e em abundância, a quaresmeira tem altíssimo potencial ornamental, sendo recomendada em projetos paisagísticos, na arborização de ruas estreitas e sob redes elétricas. Além disso, apresenta grande capacidade de se adaptar, sobreviver e se desenvolver no local do plantio, sendo por estes motivos, muito utilizada na arborização urbana (Lorenzi, 2002).

Suas sementes são minúsculas, dificultando a coleta e manuseio, além de existir grande número de sementes abortadas e com baixa germinabilidade (Elisson et al., 1993; Barroso et al., 1999). Assim, faz-se necessária a utilização de meios mais eficientes de propagação.

A propagação vegetativa pode ocorrer de diversas formas, bastando-se promover a regeneração de uma parte qualquer retirada de uma plantamatriz a fim de formar um novo indivíduo por meio da indução do enraizamento adventício (Janick, 1966; Hartmann et al., 2002). Uma das formas de propagação vegetativa é a estaquia, a qual permite a obtenção de um grande número de mudas com alta uniformidade a partir de uma planta-matriz, além de antecipar o período de florescimento por reduzir o período juvenil da mesma (Hartmann et al., 2002).

Para que a estaquia tenha sucesso, é necessário que ocorra a formação de raízes adventícias. 0 principal hormônio vegetal envolvido no enraizamento é a auxina. A aplicação de auxinas exógenas em estacas estimula a formação de raízes adventícias, aumentando a porcentagem de estacas enraizadas e o número de raízes por estaca (Hartmann et al., 2002), sendo que o ácido indolbutírico (AIB) é a auxina sintética mais utilizada devido a sua baixa mobilidade, maior estabilidade química e, quando aplicado em concentrações adequadas, não ser fitotóxico (Audus, 1963)

O presente trabalho teve como objetivo desenvolver um protocolo de enraizamento de Tibouchina fothergillae (D. C.) Cogn. pela aplicação de diferentes concentrações de ácido indolbutírico aplicadas via talco.

\section{MATERIAL E MÉTODOS}

Foram coletados ramos semilenhosos de uma planta-matriz localizada nos jardins do Centro Politécnico da Universidade Federal do Paraná, na cidade de Curitiba-PR, em 15 de abril de 2011.

Foram confeccionadas estacas semilenhosas de $8 \mathrm{~cm}$ de comprimento, com corte em bisel na parte inferior e corte reto na parte superior, mantendo duas folhas na porção apical com sua área reduzida à metade.

As estacas foram submetidas à desinfestação com hipoclorito de sódio $0,5 \%$ por 10 minutos, sendo posteriormente lavadas em água corrente por 5 minutos. Na base das estacas foram aplicados os tratamentos com ácido indolbutírico (AIB) veiculado na forma de talco, nas concentrações de 0, $1000 \mathrm{e}$ $2000 \mathrm{mg} \mathrm{Kg}^{-1}$ de AIB, as quais foram plantadas em tubetes de polipropileno de $53 \mathrm{~cm}^{3}$, com vermiculita de granulometria média e casca de arroz carbonizado na proporção de 1:1 como substrato, sendo então levadas para casa de vegetação com nebulização intermitente.

Após 60 dias, verificou-se a porcentagem de enraizamento, número de raízes por estaca, comprimentos das três maiores raízes por estaca, porcentagem de estacas com calos, vivas e mortas.

$O$ delineamento experimental foi inteiramente casualizado, com 5 repetições de 20 estacas por unidade experimental. As variâncias dos tratamentos foram testadas quanto à homogeneidade pelo teste de Bartlett. Aquelas que se mostraram homogêneas foram submetidas à análise de variância $e$ comparadas pelo teste de Tukey ao nível de 5\%.

\section{RESULTADOS E DISCUSSÃO}

As variáveis porcentagem de enraizamento, número de raízes por estaca, comprimento das três maiores raízes por estaca e porcentagem de estacas mortas se mostraram homogêneas e foram submetidas à comparação de médias pelo teste de Tukey ao nível de $5 \%$ de probabilidade.

Foram verificadas médias de enraizamento de $92 \%$ para os tratamentos com $0 \mathrm{mg} \mathrm{Kg}^{-1}$ e $2000 \mathrm{mg}$ $\mathrm{Kg}^{-1}$ e $87 \%$ para o tratamento com $1000 \mathrm{mg} \mathrm{Kg}^{-1}$ (Tabela 1), não sendo esta diferença significativa. $\mathrm{O}$ sucesso de enraizamento da $T$. fothergillae foi observado também por Mayer et al. (2003), tratando estacas semilenhosas com 0, 2000, 4000 e 8000 $\mathrm{mg} \mathrm{L}^{-1}$ de AIB em solução, obtendo $100 \%$ de enraizamento para todos os tratamentos com exceção da aplicação de $8000 \mathrm{mg} \mathrm{L}^{-1}$ de AIB $(89,6 \%)$. Bortolini et al. (2005) observaram $100 \%$ de enraizamento tratando estacas caulinares de $T$. fothergillae com 0 , 1500 e $3000 \mathrm{mg} \mathrm{L}^{-1}$ de AIB, enquanto Ribeiro et al. (2007) observaram enraizamento de $94 \%$ das estacas testemunhas de $T$. fothergillae. Essa facilidade de enraizamento pode estar relacionada à presença satisfatória de auxina endógena na planta e à presença de folhas nas estacas, que servem como fontes de auxinas, carboidratos e outras substâncias (Hartmann et al. 2002).

Para o número de raízes por estaca, foram observadas médias de 7,46 para o tratamento com $0 \mathrm{mg} \mathrm{Kg}^{-1}, 9,23$ para o tratamento com $1000 \mathrm{mg} \mathrm{Kg}^{-}$ 1 e 7,57 para o tratamento com $2000 \mathrm{mg} \mathrm{Kg}^{-1}$ (Tabela 1). Bortolini et al. (2005) encontraram maior número de raízes para todos os tratamentos, tendo 22,41 raízes no tratamento testemunha. As médias de comprimento das 3 maiores raízes por estaca foram de $5,87 \mathrm{~cm}$ para o tratamento com $0 \mathrm{mg} \mathrm{Kg}^{-1}$, $5,09 \mathrm{~cm}$ para o tratamento com $1000 \mathrm{mg} \mathrm{Kg}^{-1}$ e 4,81 $\mathrm{cm}$ para o tratamento com $2000 \mathrm{mg} \mathrm{Kg}^{-1}$ (Tabela 1). 
NICKNICH, R., et al. Influência do ácido...

TABELA1 - Porcentagem de estacas semilenhosas de Tibouchina fothergillae (D. C.) Cogn. enraizadas (EE), número médio de raízes por estaca (NRE), comprimento médio das 3 maiores raízes por estaca $(C M R)$, porcentagem de estacas com calos (EC), vivas (EV) e mortas (EM). Curitiba - PR, 2011

\begin{tabular}{ccccccc}
\hline AIB $\left(\mathrm{mg} \mathrm{Kg}^{-1}\right)$ & EE $(\%)$ & NRE & CMR $(\mathrm{cm})$ & EC $(\%)$ & EV (\%) & EM (\%) \\
\hline 0 & $92,0 \mathrm{a}$ & $7,5 \mathrm{a}$ & $5,9 \mathrm{a}$ & 0 & 3,0 & $5,0 \mathrm{a}$ \\
1000 & $87,0 \mathrm{a}$ & $9,2 \mathrm{a}$ & $5,1 \mathrm{a}$ & 0 & 3,0 & $10,0 \mathrm{a}$ \\
2000 & $92,0 \mathrm{a}$ & $7,6 \mathrm{a}$ & $4,8 \mathrm{a}$ & 0 & 0 & $8,0 \mathrm{a}$ \\
\hline Média geral & 90,3 & 8,1 & 5,3 & 0 & 2 & 7,7 \\
\hline Coeficiente de varia- & 9,3 & 19,6 & 22,4 & - & - & 114,8 \\
\hline ção (\%) &
\end{tabular}

Médias seguidas pela mesma letra na coluna não diferem estatisticamente pelo teste de Tukey ao nível de $5 \%$ de probabilidade.

A porcentagem de estacas mortas foi baixa e dentro da normalidade dos padrões para a estaquia, sendo vertificadas médias de $5 \%$ para o tratamento com $0 \mathrm{mg} \mathrm{Kg}^{-1}, 10 \%$ para o tratamento com 1000 $\mathrm{mg} \mathrm{Kg}^{-1}$ e $8 \%$ para o tratamento com $2000 \mathrm{mg} \mathrm{Kg}^{-1}$ (Tabela 1). A presença de estacas mortas pode ter sido causada pelo baixo grau de lignificação do material utilizado, causando uma maior perda de água e consequentemente, morte das estacas, sendo este o motivo atribuído por Nachtigal et al. (1994) para a alta mortalidade de estacas semilenhosas de araçazeiro. Outra explicação possível foi o longo tempo em casa de vegetação que pode ter favorecido a desidratação das estacas, visto que Bortolini et al. (2005) obtiveram 100\% de enraizamento com apenas 30 dias de experimento em casa de vegetação.

Não houve formação de calos em nenhum tratamento e a porcentagem de estacas vivas (sem raízes e sem calos) foi de $3 \%$ para os tratamentos com 0 e $1000 \mathrm{mg} \mathrm{Kg}^{-1}$ de AIB, não ocorrendo estacas vivas no tratamento com $2000 \mathrm{mg} \mathrm{Kg}^{-1}$ de AIB, uma vez que a maioria das estacas enraizaram e uma pequena porcentagem morreu.

\section{CONCLUSÕES}

Nas condições em que o experimento foi realizado, pode-se concluir que $T$. fothergillae é uma espécie de fácil enraizamento. O uso de AIB não é recomendado, visto que o tratamento testemunha apresentou alta porcentagem de enraizamento.

\section{REFERÊNCIAS}

1. $\quad$ AUDUS, L.J. Plant growth substances. 2 ed. Intersciences, New York, 1966

2. BARROSO, G. M. et al. Frutos e sementes: morfologia aplicada à sistemática de dicotiledôneas. Viçosa - Imprensa Universitária, 1991, v. 2. 377 p.

3. BORTOLINI, M. et al. Propagação de quatro espécies do gênero Tibouchina Aubl. (Melastomataceae Juss.) In: CONGRESSO NACIONAL DE BOTÂNICA, 56., 2005, Curitiba. Resumos... Curitiba: Digittal Solutions, 2005. CD-ROM.

4. CÉZAR, T.M.; SOUZA, F.C.; MACIEL, R.T.; DEMBISKI, W.; ZUFFELLATO-RIBAS, K.C.; RIBAS, L.L.F.; KOEHLER, H.S. Estaquia e alporquia de Tibouchina fothergillae (D.C.) Cogn. (Melastomataceae) com a aplicação de ácido naftaleno acético. Scientia Agraria, v. 10, p. 463-468, 2009.

5. ELISSON, A.M. et al. Seed and seedling ecology of neotropical Melastomataceae. Ecology, v. 74, n. 6, p. 17331749, 1993.

6. HARTMANN, H.T.; KESTER, D.E.; DAVIS JÚNIOR, F.T.; GENEVE, R.L. Plant propagation: principles and practices 7 ed. New York, Englewood Clips, 2002, 880 p.

7. JANICK, J. A ciência da horticultura. Rio de Janeiro; F. Bastos, 1966, 485 p.

8. LORENZI, H. Árvores brasileiras: manual de identificação e cultivo de plantas arbóreas nativas do Brasil. v. 2, 2 ed. Nova Odessa: Instituto Plantarum, 2002. 384 p.

9. MAYER, J.L.; ZUFFELLATO-RIBAS, K.C.; BONA, C.; RIBAS, L.L.F.; CARPANEZZI, A.A.; TAVARES, F.R. Enraizamento e anatomia de estacas de estacas de Tibouchina fothergillae (DC.) Cogn. (Melastomataceae). In: IX CONGRESSO BRASILEIRO DE FISIOLOGIA VEGETAL, Atibaia, 2003. Caderno de resumos. Atibaia: Brasilian jornal of Plant Physiology, 2003. 148 p.

10. RIBEIRO, M.N. de O.; PAIVA, P.D.O. de., SILVA, J. da. C.B.; PAIVA, R. Efeito de ácido indolbutírico sobre estacas apicais e medianas de quaresmeira (Tibouchina fothergillae Cogn.). Revista Brasileira de Horticultura Ornamental, v.13, n.1, p. 73-78, 2007.

11. SILVA, C. V.; AFFONSO, P. Levantamento de Tibouchina AUBL. (Melastomataceae) no parque estadual da serra do mar - núcleo Curucutu - São Paulo. Revista do Instituto Florestal, v. 17, n. 2, p. 195-206, 2005. 